\title{
Dependencia y psicopatología en deportistas: un estudio piloto
}

\section{Dependence and psychopathology in athletes: a pilot study}

Fecha de recepción: 02/10/2016

Fecha de aceptación: 10/10/2016

\author{
Cristina Ortiz García \\ Universidad de Huelva \\ Graduada en Psicología. Máster en Psicología del Deporte y la \\ Actividad Física. \\ Félix Arbinaga Ibarzabal \\ Universidad de Huelva \\ Departamento de Psicología, Facultad de Ciencias de la Educación, \\ Universidad de Huelva, Campus "El Carmen".
}

\section{resumen/abstract:}

La dependencia al ejercicio físico presenta características similares a los trastornos por dependencia a sustancias (Arbinaga, 2004; Arbinaga y Cantón, 2013). El objetivo propuesto fue llevar a cabo un estudio piloto y analizar la existencia diferencial de psicopatología según el nivel de dependencia al ejercicio en una muestra de 50 deportistas. Los instrumentos utilizados fueron la "Escala de Dependencia del Ejercicio (EDS-R)" de Downs, Hausenblas y Nigg (2004) y el SCL-90-R (Derogatis, 1975). En la EDS-R no existen diferencias entre sexos y sólo aquellos que dicen tener un entrenador obtienen puntuaciones superiores, en la sub-escala Tiempo-Dedicado, frente a los que no lo tienen. Los no federados, frente a los federados, obtienen puntuaciones más altas Falta de Control y Efectos Deseados, así como una mayor puntuación en Ansiedad-Fóbica y Psicoticismo. Tras el ANOVA son los Dependientes, frente a los Sintomáticos No Dependientes y los Asintomáticos, quienes obtienen mayores puntuaciones en todas las subescalas del SCL-90-R, salvo en Hostilidad y en la subescala Fóbicos sólo se presentan diferencias entre Dependientes y Asintomáticos. En conclusión, los dependientes muestran una mayor psicopatología y sería recomendable potenciar futuros estudios con muestras más amplias para ahondar en estas relaciones.

Exercise dependence presents similar characteristics to substance dependence disorders (Adams, Millar y Graus 2003). The main goal suggested was to make a pilot study and to analyze the differentiating existence of psychopathology according to the level of exercise dependence in a sample of 50 athletes. The instruments used were the "Exercise Dependence Scale (EDS-R)" by Downs, Hausenblas and Nigg (2004), and the SCL-90-R (Derogatis, 1975). In EDS-R there are not sexual differences and just those who have a coach score higher, in Time-Dedicated subscale, in contrast to those who do not. Non-federal athletes, against federal ones, score higher in Lack of Control and Intentional Effect subscales, as well as in Anxiety-Phobic and Psychoticism. In ANOVA (EDS-R and SCL-90) Dependents are those, in contrast to Symptomatic Non-Dependents and the Asymptomatic ones, who get the highest scores in all subscales of SCL-90-R except for Hostility subscale and there are just differences among Dependents and Asymptomatic groups in Phobic subscale. In conclusion, dependents show higher levels of psychopathology and it would be recommended to strengthen future studies with larger samples to deepen these relationships.

\section{palabras clave/keywords:}

Dependencia, ejercicio, psicopatología, deportistas.

Dependence, exercise, psychopathology, athletes 


\section{Introducción}

Hoy día, la sociedad está bastante concienciada de la importancia de realizar actividad físico-deportiva de una manera continuada que los efectos positivos del ejercicio van más allá del plano meramente físico (Petruzzello, Landers, Hatfield, Kubitz y Salazar, 1991). No obstante, cabe plantearse: ¿es siempre positiva la realización de ejercicio físico? ¿Qué ocurre cuando se comienzan a traspasar los límites de la mera realización de ejercicio físico con fines saludables o competitivos para convertirlo en una actividad de carácter ritualista y de obligado cumplimiento?

La práctica excesiva de ejercicio a un nivel que puede causar problemas en aquellos que lo practican, es un comportamiento que, lejos de parecer algo excepcional, se da cada vez con mayor asiduidad. Desde la Psicología se ha bautizado a este fenómeno como dependencia o adicción al ejercicio físico y se estima que su prevalencia gira en torno al 3\% en la población general (Sussman, Lisha y Griffiths, 2011); sin embargo, puede hablarse de un aumento de la misma cuando hablamos de profesionales del mundo deportivo.

Existe evidencia de que la dependencia al ejercicio físico puede llegar a constituir una alteración relevante en la vida de la persona, no solo en su plano meramente físico sino también, en el psicológico y social (Hausenblas y Simons, 2002a). Para la consideración del ejercicio físico en exceso como dependencia al mismo, se han establecido determinados criterios operativos entre los que destacan la preocupación excesiva por el ejercicio, llegando a general rituales de obligado cumplimiento o estereotipos; sintomatología abstinente si se impide la realización de dicha actividad en forma de alteraciones del estado de ánimo, insomnio o irritabilidad; preocupación que causa malestar clínicamente significativo en diversas áreas de la vida del sujeto y que esta preocupación excesiva, no se explique mejor por otro trastorno o alteración (Arbinaga y Caracuel, 2007).

Ruiz-Juan, Zarauz y Arbinaga (2013) han llevado a cabo revisiones sobre la literatura existente al respecto y se ha encontrado que, en una población más profesionalizada en el mundo del deporte, la prevalencia llega a situarse en el 6.9\%, (Szabo y Griffiths, 2007) e incluso muy superiores y cercanos al $30.4 \%$ en grupos de triatletas (Blaydon y Lindner, 2002). Este fenómeno ha sido también estudiado entre la población universitaria. En este grupo poblacional Hausenblas y Downs (2002b) estimaron una prevalencia de la dependencia al ejercicio físico entre $3.4 \%$ y $3.14 \%$ mientras que estudios posteriores señalan que hasta un $6 \%$ de los participantes, podrían ser catalogados con rasgos de dependencia al ejercicio físico (Reche, Martínez-Rodríguez y Ortín, 2015).

En cuanto a determinadas variables sociodemográficas, González y Sicilia (2012) estudiaron la existencia de diferencias significativas en función del sexo, la edad o de si las actividades eran dirigidas en población española que acudía a gimnasio o centros de acondicionamiento físico. Los resultados señalaron una sintomatología dependiente mayor en los hombres y una puntuación de dependencia significativamente menor en las actividades físico-deportivas que son dirigidas frente a las semidirigidas o libres. Sin embargo, no todos los datos apoyan estos resultados; así Reche, Martínez-Rodríguez y Ortín (2015) concluyeron que no existían diferencias significativas en cuanto al sexo o la modalidad de ejercicio 
físico practicada. En cambio, si observaron diferencias estadísticamente significativas en cuanto al tiempo de entrenamiento, ya que encontraron mayores síntomas de dependencia en aquellos participantes que presentaban una alta dedicación.

A grandes rasgos, parece ser que las estimaciones realizadas sobre la prevalencia de la población que pudiera presentar el trastorno de la dependencia al ejercicio físico, señalan una amplia variabilidad (Arbinaga y Caracuel, 2007) hecho que podría verse precipitado, como apuntan Márquez y de la Vega, (2015) por la existencia y uso de diferentes cuestionarios de evaluación, entre otros factores.

A pesar de que se trata de un trastorno conocido desde hace varias décadas, su ubicación nosológica no está del todo aclarada (Antolin, de la Gándara y García, 2013; Arbinaga y Cantón, 2013), el debate gira en torno a dos posturas: aquellos que lo sitúan dentro de los Trastornos Adictivos (Veale, 1987), y aquellos que lo ubican dentro del Trastorno Obsesivo Compulsivo (Yates, 1991) y tampoco se encuentra recogido aún, en los manuales diagnósticos como por ejemplo el DSM-5. Al respecto, si contempla una nueva actualización que supone un cambio importante: la inclusión de adicciones conductuales (Márquez y de la Vega, 2015) donde en un futuro, podría quedar recogido este trastorno.

Los deportistas que desarrollan dependencia al ejercicio físico, se caracterizan por un empleo excesivo de su tiempo en la actividad, dejando de lado otros aspectos importantes como el trabajo o la familia; realizan ejercicio aumentando considerablemente la frecuencia y/o intensidad del mismo (Veale, 1995) y presentan una preocupación persistente por realizar esta actividad que se incrementa cuando se ve interrumpida o impedida hasta el punto de llegar a desarrollar síntomas similares a los del síndrome de abstinencia, como ansiedad, irritabilidad o depresión (Hausenblas y Downs, 2002)

Una posible explicación al porqué la realización de ejercicio excesivo podía llegar a ocasionar una conducta dependiente (Adams y Kirkby, 1998) podría estar relacionada con los procesos de recompensa psicológica. La práctica de ejercicio se relaciona, no sólo con una mejora del estado físico del organismo, sino que, además, sus beneficios repercuten en numerosas variables psicológicas (Arruza, Arribas, Gil De Montes, Irazusta, Romero y Cecchini, 2008; Hassmén, Koivula y Uutela 2000; Pérez y Devís 2003; Ramirez, Vinnacia y Suárez 2004); como, por ejemplo, se ha comprobado que ayuda a mejorar el estado de ánimo en tanto que disminuye los niveles de depresión o ansiedad (Biddle, 1992). A ello hay que sumarle los beneficios a nivel social puesto que proporciona más oportunidades para relacionarse socialmente; por lo que todo ello, suponen una serie de factores de recompensa que potencien la realización de ejercicio de manera continuada o excesiva (Pierce, Daleng, y McGowan, 1993).

Cabe pensar que, desarrollar dependencia al ejercicio físico supone un factor de riesgo importante para las personas ya que pueden llegar a manifestar alteraciones psicológicas o psicopatológicas, que disminuyan su rendimiento deportivo y se vean perjudicadas en otros ámbitos de su vida (Araya y Campos, 2007). Se crea así, un círculo vicioso que surge de la necesidad imperiosa de hacer ejercicio, la dificultad de alcanzar los límites y la recaída en problemas de tipo social, laboral o psicológico de costoso tratamiento (Antolin, De La 
Gándara y García, 2009).

Todo ello recuerda en cierto modo, a lo que acontece en el caso de la dependencia a las sustancias ya que, no sólo se han utilizado sus criterios diagnósticos como referencia para la dependencia al ejercicio físico (Adams, Millar y Graus 2003); sino que, además, las consecuencias colaterales derivadas son muy similares. Es por tanto que resulta viable pensar que el desarrollo de una conducta dependiente al ejercicio puede ir ligada a otra sintomatología de corte psicopatológico que puede llegar a traducirse, como ocurre en la adicción a las sustancias, en un caso de psicopatología dual: la existencia, ya sea de forma simultánea o secuencial, a lo largo de la vida de un trastorno adictivo y otro trastorno mental (Szerman y Martinez-Raga, 2015) como por ejemplo trastornos de ansiedad (Sáiz , Jiménez, Díaz, García-Portillo, Marina, Al-Halabi, Szerman, Bobes y Ruiz, 2014), depresión (Salazar, Caballero, Carballoso, y Mendoza, 2014), psicosis (Szerman, 2015) o juego patológico (van der Maas, 2016).

Se han realizado encuestas epidemiológicas a gran escala en población general (Degenhardt, Chiu, Sampson, Kessler y Anthony, 2007; Grant et al., 2015; Kessler, Crum, Warner, Nelson, Schulenberg y Anthony, 1997; Regier et al., 1990) para valorar el fenómeno de la psicopatología dual y todas, han coincidido en apuntar que la ocurrencia de trastornos duales es más frecuente de lo que se piensa (Vega et al., 2015). Estudios epidemiológicos a nivel internacional han puesto de manifiesto que la morbilidad entre la dependencia a sustancias gira en torno al $70 \%$ y $80 \%$ en pacientes con esquizofrenia (Westermeyer, 2006), supera el 60\% en pacientes bipolares (Regier et al., 1990); más del 70\% en pacientes con trastornos de personalidad (Verheul, 2001) y más del 30\% en trastornos depresivos o de ansiedad (Comptom, Thomas, Stinson, y Grant, 2007) o Trastorno por Déficit de Atención con Hiperactividad (Wilens, 2007). Uno de los estudios más relevantes en el panorama nacional sobre la prevalencia de la psicopatología dual en España fue el realizado por la Sociedad Española de Patología Dual (SEPD) (Arias et al.,2013). En dicho estudio, se valoró la prevalencia de este fenómeno en una muestra de 837 sujetos de la red de Salud Mental y de la red de Drogodependencias de la Comunidad de Madrid. Los resultados revelaron que un $61.8 \%$ de los pacientes presentaba una psicopatología dual y que los casos más diagnosticados eran de trastorno bipolar, agorafobia, trastorno de ansiedad generalidad, trastornos de la personalidad, estrés postraumático y un mayor riesgo de suicidio en estos pacientes.

La relación entre dependencia al ejercicio físico y psicopatología supone un fenómeno novedoso, dada la escasa existencia de estudios previos, aunque si se han encontrado trabajos donde se aborda la prevalencia de determinadas psicopatologías en muestras de deportistas (De Francisco, Garcés de los Fayos y Arce, 2014; Dosil y Díaz, 2012; Duran, Jiménez, Jiménez, y Camacho, 2006). Uno de los más relevantes fue el de Recio y Cortés (1997) quienes estudiaron la prevalencia de los trastornos psicopatológicos en una muestra de 1376 deportistas españoles de alto rendimiento. Los resultados indicaron que las psicopatologías más prevalentes fueron los trastornos de la conducta alimentaria (con una prevalencia del $7 \%$ en bulimia y del $1 \%$ en anorexia), los trastornos del sueño (prevalencia del $4 \%$ en insomnio, $2 \%$ en sonambulismo e hipersomnio y del $1 \%$ en terrores nocturnos) y los trastornos adaptativos (prevalencia de del 3\%). Por otro lado, los cuadros psicopatológicos que 
no llegan a constituir un trastorno en sí, pero que aumentan el riesgo de padecerlo, fueron los síndromes depresivos (cuadros depresivos reactivos $1.52 \%$ y síndrome depresivo ansiógeno $0.79 \%$ ) y los del comportamiento alimentario (con un porcentaje de 0.21 de cuadros anorexiformes y un $0.58 \%$ de cuadros bulimiformes). Por último, en otras publicaciones, se apunta a una mayor prevalencia de trastornos ansiosos o afectivos en mujeres mientras que en hombres, es mayor la prevalencia de trastornos bipolares y psicóticos (Miquel, Roncero, López- Ortiz, y Casas, 2011).

Por tanto, el objetivo de la presente investigación fue llevar a cabo un estudio piloto y analizar la existencia diferencial de psicopatología según el nivel de dependencia al ejercicio en personas que practican deporte individual. Se abre así paso, a una nueva línea de estudios con la que, por un lado, se pretende servir de inspiración a otros autores (dada la escasez de estudios hasta el momento) y por el otro, responder a la necesidad de ahondar en las posibles consecuencias psicopatológicas que se pudieran llegar a derivar del desarrollo de una dependencia al ejercicio físico puesto que ello, supondría un factor de bastante peso en cuanto al rendimiento deportivo de los individuos y por ende, a su calidad de vida así como a su bienestar, no solo físico sino también, psicológico.

\section{Método}

\section{Participantes}

La muestra estuvo compuesta por un total de 50 personas, de las cuales $41(82 \%)$ eran hombres y 9 (18\%) mujeres (tabla 1). La edad media de la muestra es 36.86 (D.T.=11.85) (Min=18 y Max=67). El 34\% practica atletismo; un 32\% ciclismo; un 14\% duatlón; un 16 $\%$ triatlón; y un $4 \%$ practicaba otras modalidades. El 46\% reconoce estar Federado y el 34\% manifiesta disponer de entrenador que dirige las sesiones.

\section{Instrumentos}

Para la recogida de datos se confeccionó una entrevista ad hoc para los datos socio-deportivos (edad, sexo, modalidad deportiva, años entrenando, federado, entrenador, duración de las sesiones de entrenamiento y kilómetros de entrenamiento semanal a nado, bicicleta o en carrera).

La dependencia al ejercicio físico se evaluó a través de la Exercise Dependence Scale-Revised, (EDS-R) de Downs, Hausenblas y Nigg (2004) en su versión adaptada al español por Sicilia y González-Cutre (2011). Este instrumento presenta unos buenos índices de ajuste en el análisis factorial confirmatoria y adecuados niveles de estabilidad temporal y consistencia interna. La EDS-R está compuesta por un total de 21 ítems, con respuestas en una escala tipo Likert que iría desde 1 (nunca) a 6 (siempre), obteniéndose una puntuación global en dependencia. A su vez, consta de siete subescalas compuestas por tres ítems cada una y de las que se obtienen una puntuación para cada uno de los siete criterios que definen la dependencia del ejercicio físico: tolerancia, abstinencia, efectos deseados, falta de control, reducción de otras actividades, tiempo y continuación a pesar de las consecuencias negativas. La prueba permite clasificar a los sujetos de la muestra en tres grupos: "Dependientes" o en riesgo de dependencia(puntuaciones 5-6 en al menos tres de siete criterios), "Sintomá- 
tico no dependiente" (puntuaciones 3-4 en un mínimo de tres criterios, o bien puntuaciones de 5-6 combinadas con puntuaciones de 3-4 en tres criterios, pero sin llegar a cumplir las condiciones de en riesgo) y "Asintomático no dependiente" (puntuaciones 1-2 en al menos tres criterios, pero sin llegar a cumplirlas condiciones de los sintomáticos no dependientes).

Para valorar la posible sintomatología psicopatológica se utilizó el Symptom Checklist-90-R $(S C L-90-R)$ (Derogatis, 1975) en su versión adaptada al español. Este instrumento es un inventario autoadministrado que permite analizar la psicopatología en tres niveles de complejidad jerárquica: un nivel global, un nivel dimensional y un nivel sintomático. Evalúa el malestar subjetivo percibido por la persona a partir de 90 síntomas que quedan agrupados en nueve escalas: hostilidad, obsesión- compulsión, depresión, sensibilidad interpersonal, ansiedad, ideación paranoide, ansiedad fóbica, psicoticismo y somatización. El instrumento permite obtener tres índices generales: Total de Síntomas Positivos (TSP) que evalúa la amplitud y diversidad de la psicopatología a partir de un cierto número de síntomas presentes; Índice General Sintomático (IGS) que evalúa intensidad del sufrimiento psíquico y psicosomático y por último el Índice de Malestar (PSDI) que indica la intensidad sintomática. En cuanto a sus propiedades psicométricas han resultado ser apropiadas con una fiabilidad de las nueve dimensiones que evalúa alcanza los valores cercanos y/o superiores a $\mathrm{r}=0.70$ en test-retest y en torno a 0.80 en análisis de consistencia interna (Derogatis 1994; Derogatis y Savitz 2000).

\section{Procedimiento}

Los requisitos para ser parte de la muestra fueron: ser mayor de edad, practicar alguna modalidad deportiva considerada de resistencia, es decir que supone mantener un determinado esfuerzo durante un periodo amplio de tiempo (nadando, corriendo o en bicicleta), llevar al menos dos años entrenando y firmar el consentimiento informado.

En primer lugar, se estableció contacto, o bien a través de vía telefónica o e-mail, con los/as presidentes/as y entrenadores/as de los diferentes clubes (elegidos por cercanía y fácil accesibilidad para los investigadores a la hora de efectuar la recogida de datos) para solicitarles su colaboración. Una vez los deportistas fueron informados se les entregaba el instrumento de evaluación junto con el consentimiento informado.

Todos los análisis fueron realizados con el programa Statistical Package for the Social Sciences (SPSS 15.0).

\section{Resultados}

En primer lugar, se procedió a la realización de un análisis de la fiabilidad mediante el alfa de Cronbach, de las pruebas EDS-R y del SCL-90-R y sus respectivas subescalas. Como puede observarse (Tabla 1), la mayoría de las subescalas presentaron buenos coeficientes de fiabilidad a excepción de las subescalas del EDS-R "falta de control" y "reducción de actividades" que mostraron valores inferiores a 0.70 .

Considerando la variable sexo (Tabla 2) pudo comprobarse que no se detectaban diferencias entre los hombres y las mujeres en ninguno de los dos cuestionarios, dependencia y psicopatología, ni en las diversas subescalas de las mismas. 
Tabla 1.- Análisis de fiabilidad de las Pruebas EDS-R y SCL-90-R y Subescalas.

\begin{tabular}{l|c|l|c}
$\begin{array}{l}\text { Escala de Dependencia } \\
\text { (EDS-R) }\end{array}$ & $\alpha$ Cronbach & $\begin{array}{l}\text { Symptom Checklist-90-R } \\
\text { (SCL-90-R) }\end{array}$ \\
\hline Abstinencia & .902 & Somatización & .974 \\
Continuidad & .914 & Obsesión-Compulsión & .825 \\
Tolerancia & .905 & Sensitividad-Interpersonal & .825 \\
Falta de Control & .676 & Depresión & .855 \\
Reducción Actividades & .549 & Ansiedad & .865 \\
Tiempo Dedicado & .795 & Hostilidad & .847 \\
Efectos Deseados & .786 & Ansiedad Fóbica & .847 \\
& & Paranoide & .858 \\
& & Psicoticismo & .706 \\
& & Índice General & .877 \\
\hline
\end{tabular}

Tabla 2.- Comparación de medias de los grupos según el sexo

\begin{tabular}{|c|c|c|c|c|c|c|c|}
\hline & \multicolumn{4}{|l|}{ Sexo } & \multirow{4}{*}{$\mathrm{t}$} & \multirow{4}{*}{ gl } & \multirow{4}{*}{ Sig } \\
\hline & \multicolumn{2}{|l|}{ Hombre } & \multicolumn{2}{|l|}{ Mujer } & & & \\
\hline & M & DT & M & DT & & & \\
\hline \multicolumn{5}{|l|}{$E D S-R$} & & & \\
\hline Abstinencia & 2.58 & 1.54 & 2.70 & 1.17 & .23 & 48 & .818 \\
\hline Continuidad & 2.33 & 1.51 & 2.93 & 1.88 & 1.02 & 48 & .314 \\
\hline Tolerancia & 4.07 & 1.49 & 3.41 & 1.39 & 1.21 & 48 & .231 \\
\hline Falta de Control & 2.85 & 1.22 & 2.56 & .83 & .68 & 48 & .502 \\
\hline Reducción Actividades & 2.23 & .97 & 2.11 & .94 & .33 & 48 & .744 \\
\hline Tiempo Dedicado & 3.54 & 1.33 & 2.92 & 1.32 & 1.27 & 48 & .212 \\
\hline Efectos Deseados & 2.13 & 1.03 & 1.26 & .50 & 1.62 & 25 & .111 \\
\hline \multicolumn{8}{|l|}{$S C L-90-R$} \\
\hline Somatización & .50 & .50 & .60 & .40 & .55 & 47 & .586 \\
\hline Obsesión-Compulsión & .61 & .56 & .41 & .33 & 1.00 & 48 & .321 \\
\hline Sensitividad-Interpersonal & .45 & .53 & .51 & .56 & .28 & 48 & .778 \\
\hline Depresión & .46 & .50 & .51 & .49 & .27 & 47 & .778 \\
\hline Ansiedad & .34 & .51 & .34 & .24 & .00 & 48 & .998 \\
\hline Hostilidad & .38 & .54 & .33 & .44 & .23 & 48 & .818 \\
\hline Ansiedad Fóbica & .18 & .47 & .11 & .23 & .43 & 48 & .669 \\
\hline Paranoide & .50 & .57 & .41 & .40 & .48 & 48 & .631 \\
\hline Psicoticismo & .31 & .50 & .21 & .27 & .56 & 48 & .577 \\
\hline Índice General & .44 & .47 & .50 & .24 & .33 & 45 & .745 \\
\hline
\end{tabular}


Respecto a la realización de sesiones de entrenamiento con o sin entrenador, aquellos que realizan sesiones dirigidas por esta figura puntúan más alto en la subescala "Tiempo Dedicado" de la prueba EDS-R. Estas diferencias respecto a los que entrenan por cuenta propia, son significativas

Si las variables dependientes son comparadas en función de si los deportistas dicen disponer de entrenador, puede comprobarse que aquellos que dicen disponer de uno obtienen una mayor puntuación en la sub-escala "Tiempo Dedicado" de la prueba EDS-R Las diferencias con respecto a los que no realizan sesiones con entrenador, son significativas $(\mathrm{p}=.003)$ y presentan un tamaño efecto alto $(d=.93)$.

Tabla 3.- Comparación de medias de los grupos según se dispone de entrenador

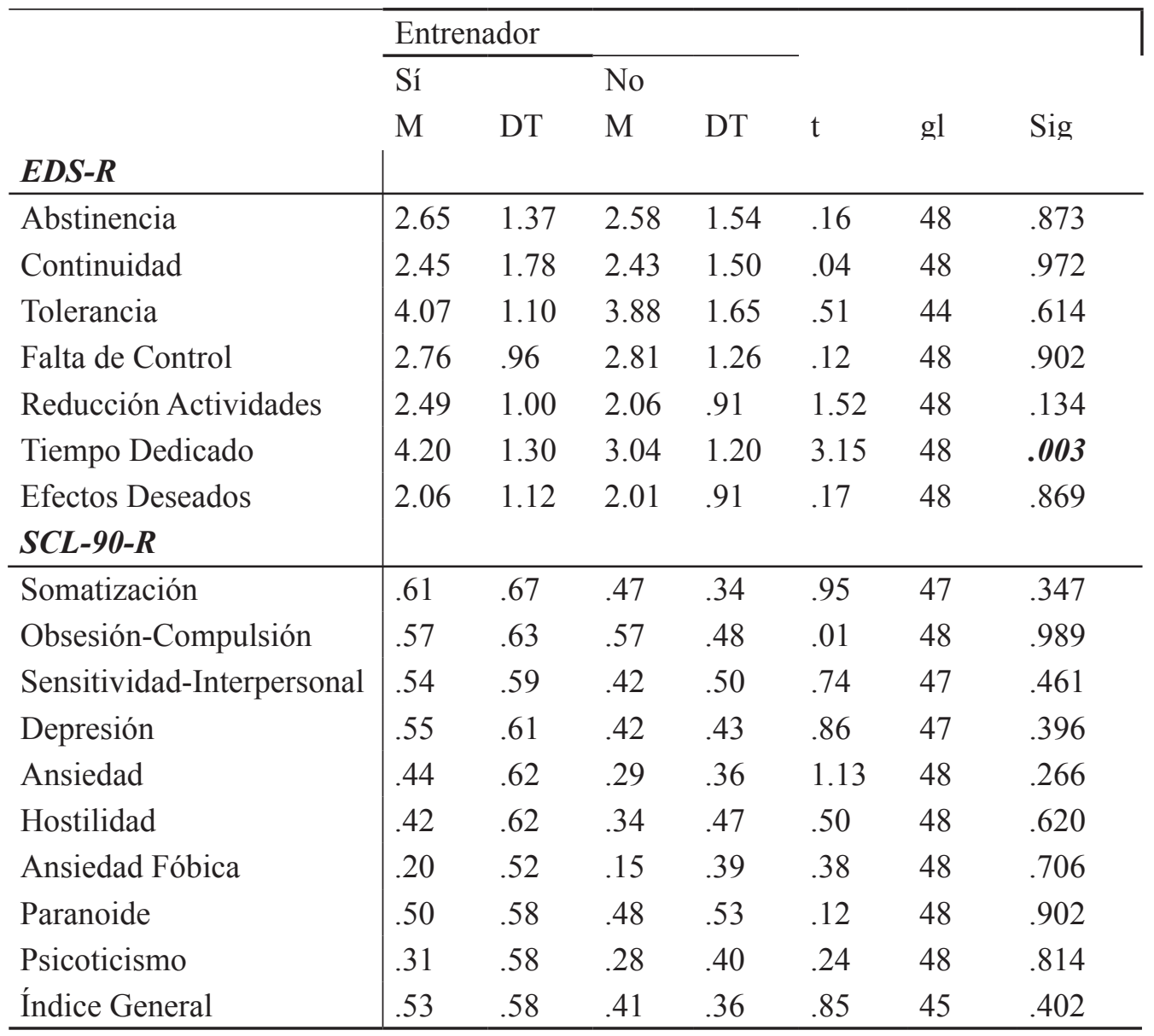


En la prueba de dependencia EDS-R, los deportistas que dicen no estar federados, frente a los que sí lo están (Tabla 4) muestran diferencias significativas en "Falta de Control" ( $\mathrm{p}=$ .006) y en la sub-escala de "Efectos Deseados" ( $\mathrm{p}=.043)$; con un tamaño efecto alto $(\mathrm{d}=.82)$ y medio $(\mathrm{d}=.60)$, respectivamente. De igual forma, se aprecian diferencias significativas con un tamaño efecto medio en las subescalas del SCL-90-R “Ansiedad Fóbica” (d=.64) y "Psicoticismo" (d=.59).

Tabla 4: Comparación de medias de los grupos según se está federado

\begin{tabular}{|c|c|c|c|c|c|c|c|c|}
\hline & \multicolumn{4}{|c|}{ Federado } & \multirow{4}{*}{$\mathrm{t}$} & \multirow{4}{*}{$\mathrm{gl}$} & \multirow{4}{*}{ Sig } & \multirow{4}{*}{ d } \\
\hline & \multicolumn{2}{|l|}{ Sí } & \multicolumn{2}{|l|}{ No } & & & & \\
\hline & M & DT & M & DT & & & & \\
\hline$E D S-R$ & & & & & & & & \\
\hline Abstinencia & 2.30 & 1.37 & 2.85 & 1.54 & 1.32 & 48 & .193 & \\
\hline Continuidad & 2.29 & 1.66 & 2.57 & 1.53 & .62 & 48 & .541 & \\
\hline Tolerancia & 4.09 & 1.50 & 3.83 & 1.48 & .62 & 48 & .541 & \\
\hline Falta de Control & 2.32 & .93 & 3.20 & 1.19 & 2.86 & 48 & .006 & .82 \\
\hline Reducción Actividades & 2.03 & .86 & 2.36 & 1.03 & 1.22 & 48 & .229 & \\
\hline Tiempo Dedicado & 3.68 & 1.34 & 3.22 & 1.33 & 1.22 & 48 & .230 & \\
\hline Efectos Deseados & 1.72 & .81 & 2.28 & 1.05 & 2.08 & 48 & .043 & .60 \\
\hline$S C L-90-R$ & & & & & & & & \\
\hline Somatización & .41 & .36 & .62 & .55 & 1.53 & 47 & .134 & \\
\hline Obsesión-Compulsión & .45 & .40 & .68 & .61 & 1.55 & 48 & .128 & \\
\hline Sensitividad-Interpersonal & .39 & .49 & .51 & .57 & .79 & 47 & .435 & \\
\hline Depresión & .41 & .41 & .51 & .55 & .73 & 47 & .469 & \\
\hline Ansiedad & .22 & .30 & .44 & .56 & 1.68 & 48 & .099 & \\
\hline Hostilidad & .28 & .30 & .45 & .65 & 1.26 & 38 & .217 & \\
\hline Ansiedad Fóbica & .03 & .06 & .29 & .57 & 2.30 & 27 & .030 & .64 \\
\hline Paranoide & .41 & .48 & .56 & .58 & .98 & 48 & .333 & \\
\hline Psicoticismo & .15 & .24 & .41 & .57 & 2.19 & 36 & .035 & .59 \\
\hline Índice General & .35 & .30 & .54 & .51 & 1.53 & 45 & .131 & \\
\hline
\end{tabular}

Finalmente, según el ANOVA realizado comparando las puntuaciones en el SCL-90-R y sus sub-escalas, en función del nivel de dependencia obtenido -Dependientes, Sintomáticos No Dependientes y Asintomáticos- en la escala EDS-R (Tabla 5) es posible observar que existen diferencias significativas entre los tres grupos en todas las subescalas del SCL-90-R a excepción de en la subescala "Hostilidad" y en la subescala "Ansiedad Fóbica" dónde no se aprecian diferencias entre el grupo Dependiente y el grupo Sintomático No Dependientes. 
En el resto de las subescalas son los Dependientes quiénes presentan puntuaciones más elevadas y significativas; a diferencia de lo que ocurre cuando se comparan el grupo Sintomáticos No Dependientes y el grupo Asintomático, donde no se aprecia diferenciación alguna.

Tabla 5.- ANOVA de las escalas EDS-R y las del SCL-90-R.

\begin{tabular}{|c|c|c|c|c|c|c|c|c|c|}
\hline & $\begin{array}{l}\text { Categorías } \\
\text { EDS-R }\end{array}$ & M & DT & $\mathrm{F}$ & gl & Sig. & $\begin{array}{l}\text { Contraste } \\
\text { de medias }\end{array}$ & Sig. & $\eta_{p}^{2}$ \\
\hline \multirow[t]{3}{*}{ Somatización } & Dependiente & 1.35 & 1.02 & 12.30 & 2 & .000 & Dep.>sint. & .003 & .35 \\
\hline & Sintomático & .59 & .32 & & & & Dep $>$ asint. & .000 & \\
\hline & Asintomático & .33 & .30 & & & & & & \\
\hline \multirow[t]{3}{*}{ Obsesión-Compulsión } & Dependiente & 1.35 & .87 & 7.94 & 2 & .001 & Dep.>sint. & .026 & .25 \\
\hline & Sintomático & .66 & .47 & & & & Dep.>asint. & .001 & \\
\hline & Asintomático & .38 & .39 & & & & & & \\
\hline \multirow[t]{3}{*}{ Sensitividad-Interpersonal } & Dependiente & 1.22 & .78 & 5.85 & 2 & .005 & Dep. $>$ sint. & .019 & .20 \\
\hline & Sintomático & .47 & .40 & & & & Dep. $>$ asint & .004 & \\
\hline & Asintomático & .33 & .49 & & & & & & \\
\hline \multirow[t]{3}{*}{ Depresión } & Dependiente & 1.29 & .89 & 8.51 & 2 & .001 & Dep. $>$ sint. & .003 & .27 \\
\hline & Sintomático & .47 & .36 & & & & Dep.>asint & .000 & \\
\hline & Asintomático & .34 & .39 & & & & & & \\
\hline \multirow[t]{3}{*}{ Ansiedad } & Dependiente & 1.13 & .97 & 9.53 & 2 & .000 & Dep. $>\operatorname{sint}$. & .004 & .29 \\
\hline & Sintomático & .39 & .39 & & & & Dep. $>$ asint & .000 & \\
\hline & Asintomático & .19 & .28 & & & & & & \\
\hline \multirow[t]{3}{*}{ Hostilidad } & Dependiente & 1.00 & 1.06 & 4.24 & 2 & .020 & Dep.-sint. & NS & .15 \\
\hline & Sintomático & .41 & .47 & & & & Dep.-asint & NS & \\
\hline & Asintomático & 2.44 & .39 & & & & & & \\
\hline \multirow[t]{3}{*}{ Ansiedad Fóbica } & Dependiente & .679 & .99 & 4.02 & 2 & .025 & Dep.-sint. & NS & .14 \\
\hline & Sintomático & .21 & .49 & & & & Dep.>asint & .021 & \\
\hline & Asintomático & .06 & .15 & & & & & & \\
\hline \multirow[t]{3}{*}{ Paranoide } & Dependiente & 1.38 & .93 & 8.46 & 2 & .001 & Dep.>sint. & .004 & .26 \\
\hline & Sintomático & .51 & .43 & & & & Dep. >asint & .000 & \\
\hline & Asintomático & .33 & .42 & & & & & & \\
\hline \multirow[t]{3}{*}{ Psicoticismo } & Dependiente & 1.00 & .96 & 7.73 & 2 & .001 & Dep. $>$ sint. & .013 & .25 \\
\hline & Sintomático & .34 & .42 & & & & Dep.>asint & .001 & \\
\hline & Asintomático & .15 & .27 & & & & & & \\
\hline \multirow[t]{3}{*}{ Índice General } & Dependiente & 1.21 & .81 & 11.05 & 2 & .000 & Dep. $>$ sint. & .003 & .33 \\
\hline & Sintomático & .50 & .33 & & & & Dep.>asint & .000 & \\
\hline & Asintomático & .29 & .29 & & & & & & \\
\hline
\end{tabular}


Como puede observarse, el tamaño efecto correspondiente a las dierentes subescalas del SCL-90-R supera el valor preestablecido para considerarse grande $\left(\eta_{\mathrm{p}}{ }^{2}=.14\right)$ encontrándose un efecto cercano o igual a este valor, en las categorías Hostilidad y Ansiedad Fóbica respectivamente y un tamaño efecto de mayor magnitud en Somatización $\left(\eta_{\mathrm{p}}{ }^{2}=.35\right)$ y en el Índice General $\left(\eta_{\mathrm{p}}{ }^{2}=.33\right)$.

\section{Discusión}

Esta investigación es un estudio piloto que analiza principalmente, la existencia de diferencias psicopatológicas en función del nivel de dependencia al ejercicio físico, en personas que practican deporte a nivel individual, es decir, aquellos d eportes en los que el deportista realiza la actividad sin necesidad de complementarse con otros o de realizar un trabajo en equipo (en este caso, natación, atletismo, ciclismo, duatlón o triatlón). Explora, además, la diferencia de puntuaciones, tanto en las subescalas de la EDS-R como en las del SCL90-R en función de otras variables tales como el sexo, el trabajo con un entrenador o por cuenta propia, o si los deportistas estaban federados o no.

A diferencia de lo encontrado por González y Sicilia (2012) y, por el contrario, de acuerdo con otras investigaciones previas (Salazar et al., 2012, Reche, Martínez-Rodríguez y Ortín, 2015) los resultados, no han mostrado diferencias significativas en función del sexo. De igual forma, tampoco se han encontrado puntuaciones diferenciales en función de si se llevaba a cabo la práctica deportiva dirigida por un entrenador o sin él, hecho que contradice otros estudios que indicaban que las puntuaciones en dependencia eran menores en actividades dirigidas frentes a las realizadas por cuenta propia (González y Sicilia, 2012); aspecto que pudiera tener relación con el tipo de deporte analizado. Respecto a si los deportistas estaban federados los resultados han mostrado una mayor puntuación en dos dimensiones de la dependencia: la falta de control y la consecución de los efectos deseados.

Los hallazgos relevantes del presente estudio, se derivan fundamentalmente del análisis de la varianza (ANOVA) realizada entre las dimensiones de la EDS-R y el SCL-90-R. Lo destacable de estos resultados, radica en que se ha encontrado diferencias significativas entre en los tres subgrupos derivados de las puntuaciones en dependencia a ejercicio de la muestra: dependientes, sintomáticos no dependientes y asintomáticos. De tal forma que, en cada una de las dimensiones que mide el SCL-90-R, el grupo de los dependientes muestra puntuaciones superiores a las de los otros dos subgrupos. La excepción se ha podido observar en dimensiones como "Hostilidad" o "Ansiedad Fóbica" dónde no se dan diferencias significativas entre el grupo Sintomático o el Dependiente.

En este sentido, es destacable mencionar que, aunque el número de publicaciones desde la Psicología del Deporte que hacen referencia a la aparición de problemas de corte psicopatológicos en deportistas ha aumentado considerablemente (Ezquerro, 2006), pocas veces suele hacerse mención expresa a dicha problemática. Más concretamente, no se ha encontrado literatura alguna respecto a la posible relación entre la dependencia al ejercicio físico y el desarrollo, ya sea simultáneo o secuencial, de otro trastorno mental. Teniendo en cuenta los resultados encontrados, tiene cabida pensar en la aparición de este fenómeno de "psicopatología dual" y por tanto, en la concurrencia de un trastorno de Dependencia al Ejercicio Físico y otro de corte psicopatológico (ansiedad, depresión...) como un hecho nada desdeñable. 
La aparición y desarrollo de cualquier trastorno de carácter psicológico en deportistas puede suponer un factor de riesgo importante a tener en cuenta en el rendimiento deportivo del atleta (Araya y Campos, 2007). Es, por tanto, muy necesario empezar a ahondar aún más en el tema en aras a prevenir su aparición o proveer al deportista de un tratamiento adecuado frente a estas manifestaciones psicopatológicas (Guerra, 2006).

Otra de las novedades de la presente investigación, aun teniendo en cuenta el pequeño tamaño muestral, es la inclusión de modalidades deportivas como el ciclismo o la natación que, previamente, no habían sido incluidas en estudios de dependencia al ejercicio físico. Las publicaciones anteriores se han llevado a cabo principalmente en corredores (Basson y Geils, 1997; Pierce, 1994; Salay, Hayaki y Napolitano, 1998; Sanz, Blasco, y Cruz, 1992; Thorton y Scott, 1995;) o fisicoculturistas (Arbinaga y Caracuel, 2007; Salazar, 2012). Así pues, aunque en el presente estudio no se ha realizado un análisis exhaustivo teniendo en cuenta los diferentes deportes, sería interesante incorporar esta acción en estudios posteriores con modalidades deportivas como las de este estudio u otras que aún no hayan sido incluidas.

En este sentido, por ejemplo, Montero (2010) afirma que la presencia del fenómeno adictivo al ejercicio, se ha podido observar también en bailarinas, halterófilos y corredores, en concreto en aquellos que participan en maratones. No obstante, apunta a que, aunque no se han encontrado estudios con ciclistas, podría ser muy probable la aparición del fenómeno de la dependencia en este colectivo deportista suelen dedicar horas y horas a montar en bicicleta de cara a las competiciones y maratones.

Una de las limitaciones a destacar de este estudio, en parte por tratarse de un estudio piloto, es sin lugar a dudas su reducido tamaño muestral $(n=50)$, ya que con ella se ve impedida la consecución de niveles de significación mucho mayores en los análisis estadísticos realizados. Siguiendo esta línea, sería interesante la realización de nuevos estudios que permitan contrastar los hallazgos aquí encontrados utilizando muestras mucho más amplias de deportistas y, sobre todo, que posibiliten ahondar más en las posibles relaciones entre los factores de la dependencia al ejercicio físico y otros trastornos mentales. De esta forma, por ejemplo, sería factible conocer cuál de estos factores de la dependencia podría predecir mejor un malestar a consecuencia de los síntomas psicopatológicos que pudieran llegar a derivarse de este trastorno.

En esta misma línea, resultaría de crucial importancia, realizar otras investigaciones utilizando diferentes instrumentos a los mencionados en el presente documento para así, poder contrastar y/o corroborar los resultados encontrados y aportar una mayor evidencia empírica a la literatura.

Como última recomendación sería interesante insistir en la formación de entrenadores y deportistas, así como de los familiares, sobre las características y consecuencias del fenómeno de la dependencia al ejercicio físico en aras a lograr un triple objetivo: detectar posible sintomatología dependiente a tiempo, discriminar entre aquellos individuos más vulnerables a su desarrollo así como derivar a posibles "dependientes" para que reciban de forma inmediata, una adecuada atención profesional e individualizada y logren así, restablecer su bienestar psicológico. 


\section{Referencias}

Adams, J.M., Millar, T.W. y Graus, R.F. (2003). Exercise dependence: diagnostic and therapeutic issues for patients in psychotherapy. Journal of Contemporary Psychoterapy Summer, 33 (2), 93-107.

Adams, J.M. y Kirkby, R.J. (2003). El exceso de ejercicio como adicción: una revisión. RET, Revista de Toxicomanías, 34,10-22.

Antolin, V., De la Gándara, J.J. y García, I. (2009). Adicción al deporte: un problema psicosocial, (SAS). Revista Psicotropía. 8, 12-14.

Araya,G y Campos,C. (2007). Psicopatología y deporte. En movimiento. 156-157. Disponible en: http://www.edufi. ucr.ac.cr/pdf/trans/Psicopatolog\%EDa\%20y\%20deporte.pdf

Arbinaga, F. (2004). Dependencia al ejercicio. Cuadernos de Medicina Psicosomática, 71,24-32.

Arbinaga, F. y Cantón, E. (2013). Dependencia al ejercicio. En F. Arbinaga y E. Cantón (ed). Psicología del deporte y la salud, Una relación compleja. (pp. 131-172) Madrid: Editorial EOS.

Arbinaga, F. y Caracuel, J.C. (2007). Dependencia del ejercicio en fisicoculturistas valorada mediante la "Escala de Adicción General Ramón y Cajal". Revista Universitas Psycologica, 6 (3), 549-557.

Arias, F.,Szerman, N.,Vega, P., Mesias, B., Basurte,I. Morant, C., Ochoa,E. Poyo, F. y Babín, F. (2013). Estudio Madrid sobre prevalencia y características de los pacientes con patología dual en tratamiento en las redes de salud mental y de atención al drogodependiente. Adicciones, 25 (1) ,190- 200.

Arruza, J. A. Arribas, S., Gil De Montes, L., Irazusta, S.' Romero, S. y Cecchini, J.A. (2008). Repercusiones de la duración de la Actividad Físico-deportiva sobre el bienestar psicológico. Revista Internacional de Medicina y Ciencias de la Actividad Física y el Deporte, 8 (30), 171-183.

Blaydon, M. J. y Lindner, K. J. (2002). Eating disorders and exercise dependence in triathletes. Eating Disorders, 10(1), 49-60.

Biddle, S. (1992). Psicología del ejercicio y calidad de vida. En: Conferencia presentada en el Congreso Científico Olímpico. Málaga.

Comptom, W.M., Thomas, Y.F., Stinson, F.S. y Grant, B.F. (2007). Prevalence, Correlates, Disability, and Comorbidity of DSM-IV Drug Abuse and Dependence in the United States.Results from the National Epidemilogic Survey on Alcoholand Related Conditions. Archives of General Psychiatry. 64, 566-76.

De Francisco, C., Garcés de los Fayos, E.J. y Arce, C. (2014). Burnout en deportistas: Prevalencia del síndrome a través de dos medidas. Cuadernos de Psicología del Deporte, 14, (1), 22-38.

Degenhardt, L., Chiu, W.T., Sampson, N., Kessler, R.C. y Anthony, J.C. (2007). Epidemiological patterns of extra-medical drug use in the United States: evidence from the National Comorbidity Survey Replication, 2001-2003. Drug and Alcohol Dependence. 90 (2-3),210-23.

Derogatis, L.R. (1975). The SCL-90-R. Baltimore: Clinical Psychometric Research.

Derogatis, L.R. (1994). SCL-90-R. Administration, Scoring and Procedures Manual (Third Edition). Minneapolis: National Computer Systems.

Derogatis, L.R. y Savitz, K.L. (2000). The SCL-90-R and Brief Symptom Inventory (BSI) in primary care. En: Maruish, M.E. (Ed.) Handbook of psychological assessment in primary care setting (pp. 297-334). Mahwah: Lawrence Erlbaum.

Dosil, J. y Diaz, I. (2012). Trastornos de Alimentación en Deportistas de Alto Rendimiento. Consejo Superior de Deportes. Ministerio de Educación y Ciencia.

Downs, S.D., Hausenblas, H.A. y Nigg, C.R. (2004). Factorial validity and psychometric examination of the Exercise Dependence Scale-Revised. Measurement in Physical Education and Exercise Science, 8(4), 183-Disponible en: http://www.tandfonline.com/loi/hmpe20\#.VVGhtY7tmkq

Duran, L.J., Jiménez, P.J., Jiménez, F. y Camacho, M.J. (2006). Trastornos de la alimentación y el deporte. Archivos de 
Medicina del Deporte, 23 (112),117-125.

Ezquerro, M. (2006). Trastornos psicológicos en el deporte. Psicología y Deporte, 461-481.

Guerra, D. (2006). Aproximación a algunos aspectos patológicos en el deporte. Revista electrónica de Psicología Social, 11. Disponible en: http://www.funlam.edu.co/revistas/index.php/poiesis/article/view/428

Grant, B.F., Goldstein, R. B., Saha, T.D., Chou, S.P., Jung, J., Zhang H., Pickering, R.P., Ruan, W.J., Smith, S.M., Huang, B. y Hasin, D. S. (2015). Epidemiology of DSM-5 Alcohol Use Disorder: Results From the National Epidemiologic Survey on Alcohol and Related Conditions III. JAMA, 72(8),757-66.

Hausenblas, H.A. y Simons, D. (2002a). Exercise Dependence: A Systematic Review. Psychology of Sport and Exercise, 3, 89-123.

Hausenblas, H. A. y Symons, D. (2002b). How much is too much? The development and validation of the Exercise Dependence Scale. Psychology and Health, 17, 387-404.

Hassmén, P., Koivula, N. y Uutela, A. (2000). Physical Exercise and Psychological Well Being: A Population Study in Finland. Preventive Medicine, 30, 17-25.

Kessler, R.C., Crum, R.M., Warner, L.A., Nelson, C.B., Schulenberg, J. y Anthony, J.C. (1997). Lifetime co-occurrence of DSM-III-R alcohol abuse and dependence with other psychiatric disorders in the National Comorbidity Survey. Archives of General Psychiatry, 54(4),313-21.

Márquez, S. y De la Vega, R. (2015). La adicción al ejercicio: un trastorno emergente de la conducta. Revista Nutrición Hospitalaria. 31(6) 2384-2391.

Miquel, L., Roncero, C., López- Ortiz, C. y Casas, M. (2011). Diferencias de género epidemiológicas y diagnosticas según eje I en pacientes con Patología Dual. Adcciones, 23 (2),165-172.

Montero, E. (2010). La dependencia del ejercicio físico. Psicología clínica y del deporte. es. Recuperado de: http:// www.psicologiaclinicaydeldeporte.es

Pérez, V. y Devís, J. (2003). La promoción de la actividad física relacionada con la salud. La perspectiva de proceso y de resultado. Revista Internacional de Medicina y Ciencias de la Actividad Física y del Deporte, 3(10), 69-74.

Petruzzello, S.J., Landers, D.M., Hatfield,B.D., Kubitz, K.A. y Salazar, W. (1991). A meta-analysis on the Anxiety Reducing Effects of Acute and Chronic Exercise: Outcomes and Mechanisms. Sports Medicine, 11, 143-182.

Pierce, E. F. (1994). Exercise Dependence Syndrome in Runners. Sports Medicine, 18, 149-155.

Pierce, E. F., Daleng, M. L. y McGowan, R. W. (1993). Scores of exercise dependence among dancers. Perceptual and Motor Skills, 76, 531-535.

Ramirez,W., Vinnacia, S. y Suárez,G.R. (2004). El impacto de la actividad física y el deporte sobre la salud, la cognición, la socialización y el rendimiento académico: una revisión teórica. Revista de Estudios Sociales. 18, 67-75.

Reche, C., Martínez-Rodríguez, A. y Ortín, F.J. (2015). Dependencia al ejercicio físico e indicadores del estado de ánimo en deportistas universitarios. Cuadernos de Psicología del Deporte, 15 (2), 21-26.

Recio, G. P., y Cortés, J. M. (1997). Prevalencia de trastornos psicológicos en deportistas españolas de alto rendimiento. Apunts: Educación física y deportes, (50), 58-63.

Regier, D.A., Farmer, M.E., Rae D.S., Locke, B.Z., Keith, S.J., Judd, L.L. y Goodwin, F.K. (1990). Epidemiological Catchment Area study, Comorbidity of mental disorders with alcohol and other drug abuse: results from the Epidemiological catchment Area (ECA) study. JAMA, 264 (19),2511-2518.

Ruiz-Juan, F., Zarauz, A. y Arbinaga, F. (2013). Validación de la Escala de Adicción al Entrenamiento (EAE) en atletas veteranos. Adicciones 25 (4), 309-320.

Sáiz, P.A., Jimenez, L., Díaz, E.M., García-Portilla, M.P., Marina, P., Al-Halabí, S., Szerman, N., Bobes, J. y Ruiz, P. (2014). Patología dual en trastornos de ansiedad: recomendaciones en el tratamiento farmacológico. Adicciones, 26 (3), 254-274.

Sanz, T., Blasco, E. Y Cruz, J. (1992) Adicción a la actividad física de fondo. Archivos de Medicina del Deporte, 9 (35), 279-286 
Salazar, Y., Caballero, A.J., Carballoso, M.R. y Mendoza, R. (2014). Patología Dual: trastornos del estado de ánimo y de ansiedad en mujeres drogodependientes. Revista del Hospital Psiquiátrico de La Habana, 11(2).

Salazar, J.A., García, S., Remicio, C. y Villamil, S. (2012). Niveles de adicción al ejercicio corporal en personas fisicoculturistas. Revista Iberoamericana de Psicología del Ejercicio y el Deporte, 17(2), 209-226.

Sicilia, A. y González-Cutre, D. (2011). Dependence and physical exercise: Spanish validation of the Exercise Dependence Scale-Revised (EDS-R). Spanish Journal of Psychology, 14 (1), 421-431.

Slay, H. A., Hayaki, J., Napolitano, M.A. y Brownell, K.D. (1998). Motivations for running and eating attitudes in obligatory versus nonobligatory runners. International Journal of Eating Disorders, 23(3), 267-75.

Sussman, S., Lisha, N. y Griffiths, M. (2011). Prevalence of the addictions: A problem of the majority or the minority?. Evaluation $y$ the Health Professions, 34, (1), 3-56.

Szabo, A. y Griffiths, M.D. (2007). Exercise addiction in British sport science students. International Journal of Mental Health and Addiction, 5, 25-28.

Szerman, N. (2015). Patología Dual en Psicosis. Revista de Patología Dual 2 (2), 1-4.

Szerman N. y Martínez-Raga, J. (2015) “Editorial”, Advances in Dual Diagnosis, 8 (2).

Thornton, E.W. y Scott, S.E. (1995). Motivation in the committed runner: correlations between selfreport scales and behaviour. Health Promotion International, 10,177-184.

Van der Maas, M. (2016). The Social Side of the Pathways Model: Examining the Mediation of Social Support on the Relationship between Psychopathology and Problem Gambling. Journal of Gambling, 32,11-27.

Veale, D. (1987). Exercise Dependence, British Journal of Addiction, 82, 735-740.

Veale, D. (1995). Does primary exercise dependence really exist. Exercise addiction: Motivation for participation in sport and exercise, 1-5.

Vega,P., Szerman, N.; Roncero, C.; Grau-López, L.; Mesías, B.; Barral, C.; Basurte- Villamor,l.;Rodríguez-Cintas, L., Martínez-Raga, J.; Piqué, N. y Casas, M. (2015). Libro blanco: Recursos y Necesidades Asistenciales en Patología Dual. Esplugues de Llobregat : Sanidad y Ediciones, S.L.

Verheul, R. (2001). Co-morbidity of personality disorders in individuals with substance use disorders. European Psychiatry, 16(5), 274-282.

Westermeyer, J. (2006). Comorbid schizophrenia and substance abuse: a review of epidemiology and course. The American Journal on Addictions, 15, 345-55.

Wilens, T.E., (2007). The nature of the relationship between attention deficit/hyperactivity disorder and substance use. The Journal of Clinical Psychiatry, 68, 4-11.

Yates, A. (1991). Compulsive exercise and the eating disorders. New York: Bruner/Mae. 